\title{
Implementing Intermittent Preventive Treatment for Malaria in Pregnancy: Review of Prospects, Achievements, Challenges and Agenda for Research
}

\author{
Godfrey Martin Mubyazi ${ }^{1,2, *}$, Pascal Magnussen ${ }^{3}$, Catherine Goodman ${ }^{4}$, Ib Christian Bygbjerg ${ }^{5}$, \\ Andrew Yona Kitua ${ }^{1}$, Øystein Evjen Olsen ${ }^{3}$, Jens Byskov ${ }^{3}$, Kristian Schultz Hansen ${ }^{6}$ and \\ Paul Bloch ${ }^{3}$
}

${ }^{I}$ National Institute for Medical Research, P.O Box 9653 Dar es Salaam, Tanzania; ${ }^{2}$ Amani Medical Research Centre, P.O Box 81 Muheza, Tanzania; ${ }^{3}$ DBL - Centre for Health Research and Development, Faculty of Life Sciences, University of Copenhagen, Denmark; ${ }^{4}$ London School of Hygiene and Tropical Medicine, London, UK; ${ }^{5}$ Institute of International Health, Immunology and Microbiology, Faculty of Life Sciences, University of Copenhagen, Denmark and ${ }^{6}$ Department of Health Services Research, Institute of Public Health, University of Aarhus, Aarhus, Denmark

\begin{abstract}
Introduction: Implementing Intermittent Preventive Treatment for malaria in Pregnancy (IPTp) with sulfadoxine-pyrimethamine (SP) through antenatal care (ANC) clinics is recommended for malaria endemic countries. Vast biomedical literature on malaria prevention focuses more on the epidemiological and cost-effectiveness analyses of the randomised controlled trials carried out in selected geographical settings. Such studies fail to elucidate the economic, psychosocial, managerial, organization and other contextual systemic factors influencing the operational effectiveness, compliance and coverage of the recommended interventions.

Objective: To review literature on policy advances, achievements, constraints and challenges to malaria IPTp implementation, emphasising on its operational feasibility in the context of health-care financing, provision and uptake, resource constraints and psychosocial factors in Africa.

Results: The importance of IPTp in preventing unnecessary anaemia, morbidity and mortality in pregnancy and improving childbirth outcomes is highly acknowledged, although the following factors appear to be the main constraints to IPTp service delivery and uptake: cost of accessing ANC; myths and other discriminatory socio-cultural values on pregnancy; target users, perceptions and attitudes towards SP, malaria, and quality of ANC; supply and cost of SP at health facilities; understaffing and demoralised staff; ambiguity and impracticability of user-fee exemption policy guidelines on essential ANC services; implementing IPTp, bednets, HIV and syphilis screening programmes in the same clinic settings; and reports on increasing parasite resistant to SP. However, the noted increase in the coverage of the delivery of IPTp doses in several countries justify that IPTp implementation is possible and better than not.

Conclusion: IPTp for malaria is implemented in constrained conditions in Africa. This is a challenge for higher coverage of at least two doses and attainment of the Abuja targets. Yet, there are opportunities for addressing the existing challenges, and one of the useful options is the evaluation of the acceptability and viability of the existing intervention guidelines.
\end{abstract}

Key Words: Malaria, antenatal-care, intermittent-treatment, pregnancy, health services.

\section{INTRODUCTION}

\section{Malaria in Pregnancy}

Globally, malaria causes 300-500 millions cases of illness each year and is the leading cause of morbidity and mortality, especially among the pregnant women and children under the age of five years (underfives) [1-4]. Of the estimated 1-3 million malaria deaths recorded globally each year, $90 \%$ occur in sub-Saharan Africa (SSA) [5, 6]. About

*Address correspondence to this author at the National Institute for Medical Research (NIMR), Centre for Enhancement of Effective Malaria Interventions (CEEMI), P.O Box 9653, Dar es Salaam, Tanzania;

E-mails: gmmubyazi@yahoo.co.uk and mubyazig@hotmail.com
30 million women in malarious areas of SSA get pregnant each year, and the majority live in relatively stable malaria transmission [7]. Among other consequences, malaria in pregnancy can cause severe anaemia, stillbirths, spontaneous abortion, neonatal mortality, low child birth-weight, child's brain damage and inter-uterine growth retardation [8-11].

\section{Intermittent Preventive Treatment of Malaria in Preg- nancy (IPTp)}

The World Health Organization (WHO) has recommended the malaria-endemic countries to implement sulfadoxine-pyrimethamine (SP) for intermittent preventive treatment for malaria during pregnancy (IPTp) as one of the 
essential services under the focused antenatal care service package [7, 12]. The recommendation specifies at least two doses to be administered, the first one during the second trimester and the second dose during the third trimester of pregnancy $[7,12]$. To ensure compliance in uptake of IPTp doses by the pregnant women attending antenatal care clinics (ANC), the guidelines emphasise on direct observed treatment (DOT) by a qualified health worker [11, 13]. Responding to WHO's call, SSA heads of state through the Abuja Declaration commonly agreed that each pregnant woman attending ANC clinics should receive two doses of IPTp during her pregnancy period and a subsidised insecticide treated net (ITN) [14-16]. While many countries have recommended two doses of SP for IPTp, there is still debate about giving three doses or more, particularly to pregnant women who book early and continue attending ANC regularly [17], and to those who are HIV positive [15, 18]. Owing to evidence that two doses of IPTp with SP are inadequate to HIV infected women [19], multiple doses are recommended, especially in the primigravidæ $[20,21]$. As detailed below, the limiting factors include low ANC attendance behaviours, fears about HIV tests, attitudes towards $\mathrm{SP}$, and growing parasite resistance to $\mathrm{SP}$, as detailed further below.

\section{Efficacy, Safety and Cost-Effectiveness of SP for Malaria Treatment}

There are fears that SP may induce side-effects in areas with high prevalence of human immunodeficiency virus (HIV) infection $[15,18]$. This being the case from a biological perspective, it should be understood that the programmatic effectiveness of an anti-parasitic drug is determined by the efficacy of that drug against the parasite and by the drug's characteristics, including affordability, availability, acceptability to the target population, and deliverability in terms of dosing requirements and incorporation into existing ANC delivery systems [4]. Alarming resistance to SP in some parts of East Africa has been reported many years ago [22-26], and this contributed to current concerns about its effectiveness for IPTp [17]. Although P.falciparum strains contribute to SP resistance [25], poor prescription and consumption practices in the health-care system lowers the effectiveness of malaria interventions, including IPTp [25, 27]. So far, there is lack of evidence on: how IPTp-SP works in areas of low and seasonal malaria transmission areas especially in areas where SP has lost its efficacy [17]; added benefit of giving more than two doses of IPTp to each pregnant woman regardless of their HIV or gravidity status, as already this has been noted of being less advantageous [28]; perceived and real benefits and acceptability of SP to the target populations given in the existence of parasite resistance to SP especially in East and Southern Africa [11, 20, 29]; the use of artemisinin-based combination therapies (ACTs) for IPTp considering costs and risks [11, 30, 31], and acceptability of SP for IPTp following replacement of SP by ACT in several countries (e.g. Tanzania) as the first-line drug for the treatment of uncomplicated malaria $[32,33]$.

\section{Objective and Scope of this Review}

ANC clinics are the most common entry points for IPTp implementation [7, 12]. IPTp implementation through community-based health workers (HWs) and traditional birth attendants (TBAs) has been considered and tried out in Malawi [34] and Uganda [35-36] as a way of increasing coverage of IPTp and compliance. Experience from these two countries has shown that there have been some notable successes. Logically, integrating IPTp into ANC structures is highly advantageous, but the effects of one on the other are often underestimated [37]. The factors influencing IPTp implementation including the operational feasibility of IPTp and use of ANC services in general may be numerous and mutually non-exclusive [17, 34, 38]. Factors such as the perceived cost, quality and benefits of $\mathrm{ANC}$, and social values on pregnancy that makes women feel discriminated and humiliated in some ways, gender relations in the society may influence the outcome, although they are rarely analysed and documented empirically partly due to methodological difficulties [37]. Understanding the socio-cultural determinants of the use of malaria preventive services including those delivered through ANC clinics and of the compliance with drug uptake by the target populations is imperative [27]. So far, studies on social perceptions of health and disease, cultural values and people's ability to cope with the existing malaria interventions in constrained socio-economic and health system contexts are inadequate [39-41]. Other authors have suggested the following:

- evaluations of malaria prevention and treatment strategies should consider the broad health service delivery contexts, including the motivation and behaviour of health service providers and the target service users [42, 43];

- research-based evidence on how the recommended intervention is implemented is crucial to enable elucidation of the specific underlying obstacles which policy decision authorities need to know in order to take appropriate policy actions [44].

This paper is a review focusing on the policy advances, constraints and challenges to malaria IPTp implementation, with emphasis on its operational feasibility in the context of health-care financing, provision and uptake, resource constraints and diverse psychosocial factors in SSA. Broad contextual factors currently affecting (or with potential effect on) women's perceptions, attendance to, and use of ANC including IPTp, as well as health service providers' attitudes, motivation and practice on ANC (and IPTp), are highlighted and discussed. Experiences from developing countries outside Africa on ANC provision, uptake and coverage similar to those of SSA, are cited, and lessons are drawn from programmes across Africa to feed into the current policy and inform the research agenda. The paper is based on peerreviewed and non-peer reviewed articles, including grey literature and official working papers published by various agencies/authorities.

\section{REVIEW METHODS}

The articles reviewed in this paper were searched from the internet, based on the application of key words to make important downloads from the PubMed. Sometimes the search was done by typing the key words in Google first. The articles reviewed were downloaded selectively based on the contents of the information displayed. If the full articles could not be accessed directly, WHO HINARI system was consulted as done by other reviewers [11]. The search words 
mostly used in relation to IPTp are: pregnancy, perceptions, malaria, treatment, chemoprevention, intermittent, and prevention, while those used for searching for the broad aspects concerning ANC are: antenatal, provision, utilisation, demand, cost, supply, quality, Africa, developing countries. The word AND was sometimes used to combine two or more search words so as to display the article(s) needed. Additional literature was obtained using email alerts from BioMed Central for online full published articles, unpublished reports (some of which are accessible online), and hard copies of some articles found in journals documented at the local libraries, particularly at the National Institute for Medical Research (Tanzania), London School of Hygiene and Tropical Medicine (UK), DBL Centre for Health Research and Development and Faculty of Health Sciences at the University of Copenhagen (Denmark). Some articles were requested directly from the original authors. Although efforts were made to ensure that all articles relevant for this review were considered without restriction on the dates of their publication, most of the articles cited have been published between 2000 and 2007. In some parts of the results section, the present paper begins by presenting an overview of the themes related to ANC or IPTp. Then, it goes down to specific issues addressing the determinants of ANC and IPTp service supply and demand, before the general discussions and conclusions.

\section{RESULTS}

\section{Accessibility, Acceptability and Practicability of ANC and IPTp}

Accessibility and coverage of malaria interventions in SSA are constrained by poverty, limited health infrastructure, ineffective drug policy regulations and other health-care market imperfections [14, 42, 45]. Strategies towards attaining the Millennium Development Goals (MDGs) through scaling-up malaria control interventions are constrained by, among other factors, weak health systems, unclear and disputed solutions weakly supported by evidence [46]. In 1996 WHO reported that at least $40 \%$ pregnant women in developing countries receive no ANC and that nearly $31 \%$ deliver at home [47]. Coverage of IPTp in SSA varies from modest to low, as it is the case with ITNs [37]. In Blantyre (Malawi), it was found that $75.7 \%$ of 391 pregnant women who still were having ANC cards at household level had received at least one dose of IPTp, but only $36.8 \%$ received the second dose [15]. In the latter country, a previous study found that $24 \%$ of the women were not prescribed SP and only $30 \%$ received at least two IPTp doses [48]. In rural Kenya, only $19.1 \%$ of 635 study participating women reported having received SP for IPTp while only $6.8 \%$ had received two doses, despite $89.9 \%$ of the women having attended ANC [49]. At the Tanzanian National Malaria Control Programme (NMCP) level, records on IPTp showed that by March 2007 the coverage of the first and second doses (based on sentinel survey of 21 districts) were $67 \%$ and $45 \%$, respectively [33].

Contextual factors play a significant role in facilitating or constraining the effectiveness of any health intervention from the operational perspective. For instance, the effectiveness of any malaria intervention depends on consumer and provider acceptance and compliance, safety, cost, integration with other interventions, local health care delivery system and the degree of combination of all these [50]. Institutionalisation of public health interventions within constrained infrastructure, other supply and demand conditions is critical to the effectiveness of such interventions, and this has raised much concern in health policy programmes targeting the services to the populations in need [34, 42, 51, 52].

\section{Accessibility Barriers}

In SSA, women's late presentation at ANC is common, with nearly $25 \%$ of them presenting for the first time in the second trimester and for the second time during the third trimester, and this has contributed to lowering the effectiveness of ANC and IPTp related services [7, 14, 15, 28, 53]. Rural residents are relatively more disadvantaged than their urban counterparts in terms of geographical and financial accessibility to ANC [12, 47], and generally the poorer women whether in rural or urban areas are more disadvantaged [7].

Travel distance has continued being reported as one of the major challenges to women's access to and utilisation of reproductive and child health $(\mathrm{RCH})$ services [54-56]. Even if the $\mathrm{RCH}$ services were provided free of charge, the long travel distance leads to additional time cost, and in many cases, financial expenditures that overburden the poorer women who ultimately are dissuaded to attend clinics for ANC or childbirth [42, 57-64]. The timing of ANC attendance is considered to increase the opportunity for the pregnant women to receive IPTp. However, a recent study in northern Tanzania found no association between early ANC attendance and uptake of IPTp, this suggesting that the efforts to foster timely attendance alone are unlikely to improve uptake of IPTp where other contextual barriers exist [32].

\section{Travel Distance to ANC Clinics by the Target Populations}

Due to delays in contacting clinics for ANC, a large proportion of SSA countries recommended four ANC visits and increased the spacing between them in conformity to WHO protocol [62]. The optimal number of ANC visits for countries with limited resources is still debated at policy and programme levels [55]. Sometimes the target ANC women show more concern with the quality of care than the number of ANC visits [62, 65]. Basically, the four ANC visits were mainly suggested in consideration of women residing far from health facilities (HFs) [12]. However, having physical access to HFs is not enough in the acute and persistent shortages of drugs, medical equipment, other basic supplies, and HWs who are humane, skilled and motivated [42, 47, 66]. HFs may be closer, but the target users bypass them by consulting the providers perceived as being more skilled for delivering better services [67]. Attendance to ANC gives the pregnant women an opportunity for accessing health education (including counselling) related to their pregnancy and their children/babies [55]. None-regular ANC clinic attendees for various reasons (including long travel distance) have limited accessibility to IPTp services in the right time such as during the early stages of pregnancy [32], including $\mathrm{RCH}$ education in general [68, 69].

\section{User Charges in General and in Relation to ANC Services}

Experience from SSA shows that real or perceived existence of user-fees is a stumbling block to pregnant women's 
contact of RCH clinics [70]. In some areas of Tanzania [71], Uganda [72], Zimbabwe [73], Nigeria [74], and South Africa [75] user-fees have discouraged pregnant women with limited cash to attend ANC clinics. IPTp services are not necessarily free of user-fee barriers [37], especially at private HFs [43, 76], as studies in several districts in Tanzania [38], Malawi [34] and Kenya, have elucidated. In Kenya, Lynm and Munguti found that even in government clinics the ANC clients were often asked to pay for SP, leading to lower uptake of the IPTp doses recommended for such clients [34].

Implementing exemption policies for $\mathrm{RCH}$ services in SSA is hampered by the low translation of the exemption guidelines into practice at HF levels. For example, in Uganda [35], Ghana [77] and Tanzania [29, 78-80], pregnant women sometimes paid formally for laboratory tests, registration and an initial consultation fee, which were perceived as a heavy burden to such women. Outside the user-fee structure, there are other informal charges and other indirect cost hindering pregnant women's health service utilisation [58]. Misunderstanding or ignorance of the user-fee structure and exemption policy contributes to lowering health service utilisation. For example, sometimes user-fee exemption guidelines are in place but the target populations do not use their opportunity to ask/claim for exemptions, as they do not know that they are eligible [81]. In Korogwe district, Tanzania, the community was unhappy with pregnant women being required to pay for some $\mathrm{RCH}$ services contrary to what was believed to be the government policy entitling such women to be among the exempted vulnerable population groups [80].

\section{Socio-Cultural Barriers}

The extent to which socio-cultural determinants of compliance with drugs also play a role in the optimal utilisation of existing HF based services has not been adequately studied, especially in a problem solving approach to improve facility utilisation and women's participation in the IPTp strategy [19]. Along with the financial barriers, it is widely evident that women in most developing countries are facing social-cultural barriers to their access to HFs delivering $\mathrm{RCH}$ services [70]. Such barriers have strong roots into gender imbalances beyond the HF settings limiting their autonomy and power to attend ANC clinics and use IPTp services [30, 37], among other RCH services [82]. Shying away or fearing to reveal one's pregnancy in the early stage is one of the barriers [37], as validated by findings from Tanzania [33]. Sometimes women delay to attend ANC clinics due to lack of permission from their spouses [47]. Studies in Tanzania (Centre for Enhancement of Effective Malaria Interventions 2006, unpublished Research Report) and The Gambia (Gates Malaria Partnership-DBL 2005, unpublished Consultancy Report) found that teenagers feared the severe legal and social implications of getting pregnant while schooling or before marriage. The two studies also found that delay in contacting ANC clinics also occurred in (a) married women who carried pregnancies outside the wedlock or did not have socially recognised partners (b) women aged from 35 years and above who shied away to be accompanied or seen by much younger pregnant women. Consequently, the pregnant women concerned did not access either one or both doses of IPTp and other ANC services.
Age may influence pregnant women prefer particular service providers. A study on the determinants of pregnant women's use of IPTp services in Mufindi district, Tanzania [38] noted some older women disliking to be attended by the nurses whose ages were as low as the ages of such women's daughters. Myths and unfound beliefs may also negatively affect ANC attendances. In Mukono district, Uganda, women delayed attending ANC clinics and lowly utilised ITNs and IPTp due to the prevailing community myth and belief that adolescent girls and primigravidæ were at low risk $[35,83]$.

\section{Provider-Client Interactions and Perceptions of Quality of} ANC in Generals

Supply and demand related factors in a general or the broad health service situation contribute influencing the target clients' choice of the service. Women may delay or completely decline to attend clinic because they appreciate comparative better service they received from the TBAs who may also portray better attitude of friendliness and devoting more time for listening to their clients about their health and social problems. Also, by experiencing frequent shortages of needed medication at ANC clinic, women find such services unreliable while they do not suffer such experience at TBAs [72]. Women are disappointed when: they wait longer at the service delivery point, are mishandled by nurses, and lack of diagnostic facilities [35, 76, 84], avoid contacting clinics for ANC in fear of the lack of privacy at the consultation or bad language of the nurses [85], face unfriendly opening hours and/or unfair and unexpected costs [56]. The need for the skilled ANC staff in the health-care system is one of the prerequisites for attracting women to clinics [56]. Moreover, lack of- or shortage of the essential utilities such as cups at ANC level for use by the patients taking SP under DOT has constrained IPTp implementation in Tanzania, Uganda and Malawi [34]. Not all the ANC clients feel comfortable when they are required to share cups when taking the drugs under DOT for IPTp [86]. Generally, analysis of trends and levels of ANC service provision in Africa and other developing countries has revealed that the ANC service provided are substandard, therefore, significantly contributing to lower the uptake of such services [12, 34, 55, 56, 62, 67]. Vast literature documents the concern raised about the factors hindering pregnant women to attend clinics, but as Fathalla [87] argues, the key question service providers must ask themselves is, "do we offer the service that the women will accept?, instead of asking "why do women not accept the service we offer?" [56].

\section{Perceptions and Acceptability of SP}

\section{Psychological Barriers: Perspective of Pregnant Women and the Society Around them Concerning SP}

Findings from SSA show pregnant women and the society associating SP with severe adverse outcomes such as abortion, skin reaction and lack of anti-fever effect $[14,30$, $34,35,80,83,88-90]$. Truly, SP can cause effects such as Stevens-Johnson syndrome in people who are allergic to sulfa with possible dramatic and potentially fatal effects [9194]. However, such side-effects are rare and in most cases exaggerated $[10,25,95]$. The perception people have on a drug may significantly determine their acceptance of that particular drug [37]. A drug that is bitter or associated with 
abortion in some cultures is likely to be less complied with by the target population [96]. The situation is worse when health service providers share such sentiments and hence are unable to provide service users with correct information about the drug. In Tanzania, some health professionals expressed their doubts about the rationale for recommending SP for malaria treatment in areas where drug resistance is already high [97]. In Malawi, some HWs believed that SP was not appropriate to be taken by a person with an empty stomach [34].

\section{Health Care Workers' Knowledge and Administration of IPTp-SP}

It is important that health-care providers know well the health problems against which the services are provided. This includes knowledge on the causes, management and prevention of such problems (including diseases like malaria), which is essential for their own motivation and confidence in managing or giving advice to their clients [98]. One study in Kenya found that IPTp was not administered at some clinics due to HWs being unaware of the guidelines [14]. Similarly in Tanzania, HWs in two districts reported confusion they faced regarding timing of the doses and the number of the doses to be administered for IPTp [33]. In this circumstance of limited knowledge among the HWs, the effectiveness and coverage of health interventions remain to be undermined, and may expose the target populations to the risk of being attended by uninformed HWs [6]. A number of malaria control programmes deliberately emphasise initial training of $\mathrm{HWs}$ in order to improve their communication skills and prevent this negative effect [34].

Health Service Providers' Morale at their Workplaces, Perceptions on SP and its Implications on ANC-IPTp

Reducing poverty by preventing malaria in pregnancy is among the MDGs [99, 100]. But serious shortage of skilled and motivated staff in SSA and other developing countries' health sectors, is a conundrum [101]. The working environment may affect HWs' morale and performance of different health interventions including IPTp. In Tanzania, Uganda and Zambia, HF understaffing contributed to HWs' perceived overwork, affecting the quality of care and HWs, adherence to IPTp-DOT guidelines [34]. Also, poor infrastructural status and low staff remuneration have demoralized HWs and lowered the general quality of care in SSA $[42,102]$. A recent review of country experiences indicated the shortage of water and cups at HFs having affected HWs' adherence to DOT for IPTp in Tanzania and Uganda [34]. Several HWs in Dar es Salaam and Mkuranga district, Tanzania, reported the inconvenience faced due to wasting time and occasionally spending their out-of-pocket money finding water for use at clinic level (Mubyazi, PhD Thesis, submitted). Most studies report the low quality of the health-care given by the nurses to discourage women in utilising $\mathrm{RCH}$ services. This is a reality, although it is important to consider the reasons for such HWs to behave/practice in the way they do rather than only accusing them. For instance, studies in Zimbabwe [103, 104] and Tanzania [84] found nurses complaining to have been working under pressure and with little sympathy from their clients.

The issue of unofficial user-fees on the fee-empted $\mathrm{RCH}$ services discussed before can be connected to health service providers' working condition. There is evidence that even when the HWs are informed of the exemption policy, they may still charge their clients due to poor remuneration and economic pressures to maintain their own good living [70]. ANC staff may also fail to administer SP not necessarily due to loss of interest, but pregnant women may hate taking SP, or due to drug shortages as noted in Tanzania [32], Kenya [14] and Malawi [34].

In areas where there is no service, outreach clinic services are crucial to ensure physical accessibility [56]. Although mobile clinics have been implemented in attempt to increase coverage of the remote and difficult-to-reach communities, they are hampered by low morale of the HWs who dislike travelling to remote places. So far evidence shows that the IPTp strategy like other drug-based malaria interventions is constrained by low outreach coverage [19]. In Korogwe district (Tanzania) district health management team (DHMT) reported lack of transport occasionally limiting the DHMT, s ability to reach remote HFs for health service supervision as planned. Therefore, it was suggested that concerted efforts for improving HWs' morale (including the issue of transport and number of HF staff) are necessary if compliance with IPTp service provision has to be realised [76].

\section{Achievements and Opportunities for IPTp-SP and ANC Services}

Despite the constraints mentioned above, IPTp implementation in SSA in SSA is happening and there are indications that it is possible to attain the Abuja target of the $60 \%$ coverage of pregnant women receiving IPTp [7, 12]. Recently, countries such as Kenya, Malawi and Tanzania have reached and even passed the $60 \%$ coverage of women attending ANC clinics for two or more times and in some districts IPTp coverage have reached $>60 \%$ for the first dose [34, 105]. There is also an encouraging trend in the coverage for the second dose although still less than 50\% in Tanzania [33], as in many other countries in southern Africa [34, 105]. The IPTp coverage records are encouraging, though not much, from a policy and programme perspective. However, good access to ANC does not warrant high uptake of IPTp-SP, since quality of care delivery factors, HWs, knowledge and motivations, and target population's knowledge, attitudes towards IPTp and practices remain important [32-34, 47].

\section{DISCUSSION}

\section{Importance of IPTp}

There has been a common appreciation on importance of malaria IPTp in SSA. However, based on different authors' observations, special consideration should be given to possible factors that may hinder the achievement of the desired level of coverage.

\section{Socio-Economic and Systemic Considerations}

\section{Service Provision/Supply Issues}

Advocacy for critical and broad analysis of factors affecting malaria interventions [42] is supported by evidence elucidated regarding the understaffing, shortage of utilities and drugs at a number of HFs. Low knowledge especially on the rationale and benefits of interventions may remain critical to 
IPTp implementation if concerted efforts are not made to overcome the challenges. It is still critical as it allows low adherence to IPTp, false beliefs and perceptions, and may even bias the performance of the HWs. Informal visits to TBA is unlikely to cease in SSA communities, and despite the need for involving the TBAs in malaria prevention strategies, such providers should consider themselves as replacement of the formal ANC system, rather than complementing it. Also, any view that the target service users are most likely to be less aware of a new intervention is not supported by evidence on the low knowledge on IPTp guidelines from a study of HWs [33-34]. This suggests that policy and programme authorities should continue considering the building the capacity of the existing HWs by allocating additional resources for training and emphasising on regular supervision and periodically monitoring their performances.

Although some of the factors identified to lead to the low HWs' morale, including understaffing, poor infrastructural facilities and unsatisfactory salaries may not be removed overnight, efforts are required towards improvement. Working on some of these problems may not only (and necessarily) need additional financial resources, but (also) an improved management system that allows responsiveness to local needs and accountability. HWs may not necessarily claim for increased salaries rather than needing timely remuneration packages.

\section{Service Demand Issues}

User-fees may remain as a crucial health care financing mechanism both in the public and private sectors. Nevertheless, their implementation without affecting $\mathrm{RCH}$ services is questioned considering resource-poor settings where already pregnant women are constrained by other social-cultural gender biased conditions [70]. It should be remembered that poverty is rampant and both the pregnant women and the HWs are under undue economic pressures. Anything that may be used as an excuse to evade the pregnant women to attend HFs implementing user-fees will sound well to such women and add to reduction of the many roadblocks towards achieving the Abuja targets. Wrong beliefs about the intervention may be minimised through equipping the HWs with current knowledge and improving their working conditions so that they can be better advocates for health interventions (including IPTp). To most of the service clients and even some health service providers, prevention benefits are not seen immediately.

Even in conditions where there might be an adequate supply of drugs, the target users may still hate taking it as reported above. The Health Belief and Behavioural Theories assert that people may only adapt a disease preventive measure if they believe that they are either susceptible to or threatened by the disease with perceived consequences, and in the case of prevention they weigh the benefits of adapting the recommended method against the undesirable risks, costs and other consequences [106, 107]. Therefore, the reported delay by women to attend clinics and their negative attitudes towards SP may also be rooted from their disregard of malaria prevention through IPTp-SP, besides other reported barriers concerning imbalanced gender relations and poverty conditions facing most women in SSA.

\section{CONCLUSION}

The implementation of IPTp in SSA is undoubtedly done in a multifaceted socio-economic and ANC resource constrained settings, this having an impact on the achievement of the Abuja targets. However difficult it might be, it is crucial to get prepared for the challenges in attempt to minimise their impacts. As long as 5t 5s acknowledged that the benefits outweigh the costs or risks, scaling-up IPTp to increase coverage is crucial, considering the severe consequences to the mother and offspring of malaria in pregnancy. Establishing and using wider evidence-base may help to arrive at informed policy and management decisions related to IPTp implementation. Currently, the evidence available regarding the determinants of ANC provision, utilisation and coverage is vast for decision makers to capitalise on the strengths and work on the existing challenges. The scaling-up of IPTp would benefit from applying knowledge acquired through research on the real situation and how to improve it. Yet, political will and taking action to provide moral and financial support on operational research are crucial. This would help monitoring what works and what does not in relation to ANC [71]. In principle, educating the general population is desirable to bring everybody at a good level of understanding, hence enhancing compliance and coverage. Education and advocacy efforts must be continuous and their emphasis must be maintained at all the times. This requires a strong health system and revealed by this review, a robust health system is required for effective implementation of the IPTp strategy. The healthcare system must be strengthened by adequate staffing levels, sharpening staff skills and motivating them by improving the general staff working conditions. IPTp programme managers and frontline implementers at district level should be aware that not all HWs on malaria control services are fully knowledgeable about the intervention strategy [33]. Special attention should therefore be paid to instil such knowledge and to monitor its implementation. Improving HWs' morale to ensure durable effects requires the training of such workers and regularly supervising them, increasing the number of HWs, improving health infrastructure and working tools, and building trust and confidence among such workers and their service users. To make an important difference, operational health service and policy research should be an integral part of the intervention programme [44]. Researchers should ensure that the translation of research evidence into systemic action requires timely conduction of research, submission of the research reports and in the form that can be easy to be used by decision makers [97].

\section{RESEARCH AGENDA}

This review suggests the need for understanding the (a) perceptions and attitudes of pregnant women, the general public and health service providers concerning IPTp-SP (b) the risk of ANC clients missing SP or getting it in a substandard way (c) effect of user-fees on general services or ANC and indirect cost e.g. travel, waiting time on IPTp delivery and coverage (d) acceptability and practice of IPTp-SP in countries which have replaced SP with ACTs as the firstline drug for uncomplicated malaria as some people may have now preferred drugs other than SP which may be seen to be no longer beneficial (f) the feasibility and viability of 
IPTp in the existence of other malaria preventive methods that are promoted and implemented in different socio- cultural and health care delivery settings [30, 108] (h) implications of HIV counselling and screening services on ANC attendances and delivery/uptake of IPTp-SP. We observe that in situations whereby women have to pay for the screening services or are stigmatised by HIV screening services at ANC clinics, the delivery of IPTp may be lowered by decreased women's attendances. This observation is supported by other authors [109] who argue that in societies where women suffer abuse, social inequality and injustice, screening them for HIV at ANC clinics has both socio-cultural and ethical implications, which means ANC attendances may be reduced. The morbidity and mortality effects of malaria in pregnancy remain to be experienced if the apparently persistent and increasing trend of P.falciparum resistance to the recommended drugs is not reversed. This is a challenge, calling for the biomedical researchers to continue investigation towards discovery of alternative more effective drugs [110$112]$.

\section{AUTHORS' CONTRIBUTIONS}

As part of his PhD study, GMM conceived the study, undertook the major review and drafted the manuscript. Invaluable comments and suggestions were made by CG and AYK. CG also provided some articles for reference. Other co-authors co-supervised GMM, s PhD study under coordination by $\mathrm{PB}$ and ICB, besides their great comments on this paper. All the authors read and approved the MS for publication.

\section{ACKNOWLEDGEMENTS}

Professor Brian Greenwood (LSHTM, UK) commented on the earlier draft of the manuscript. Also, useful advice was obtained from Prof. Wen L. Kilama (AMANET, Tanzania) and Drs. Don de Savigny (STI, Switzerland) and Jasper N. Ijumba (University of Dar es Salaam, Tanzania). The study was funded by the Bill and Melinda Gates Foundation through Gates Malaria Partnership (GMP) coordinated by the London School of Hygiene and Tropical Medicine (UK) in collaboration with DBL-Centre for Health Research and Development, University of Copenhagen (Denmark), and NIMR (Tanzania). All the authors whose papers have been cited as reference materials in this paper.

\section{REFERENCES}

[1] Brabin BJ. An analysis of malaria in pregnancy in Africa. Bull WHO 1983; 61: 1005-16.

[2] Menendez C. Malaria during pregnancy: a priority area of malaria research and control. Parasitol Today 1995; 11(5): 178-83.

[3] Steketee RW, Nahlen BL, Parise ME, Menendez C. The burden of malaria in pregnancy in malaria-endemic areas. Am J Trop Med Hyg 2001; 64 (Suppl 1-2): 28-35.

[4] WHO. The use of antimalarial drugs: chemoprophylaxis treatment of malaria in special groups. WHO; 2001 [cited 2007 Jan 12]. Available from: http://www.rbm.who.int/cmc_upload/0/000/014/ 923/am_3.htm\#p3.1).

[5] Rugemalila JB, Wanga CL, Kilama WL. Sixth African Malaria day in 2006: how have we come after the Abuja Declaration? Malar J 2006; 5: 102.

[6] Snow RW. The invisible victims: A special supplement Issue. Nature 2004; 430: 934-5.

[7] WHO, UNICEF. Africa Malaria Report on Malaria in Pregnancy. World Health Organization, Geneva; 2003 [cited 2007 June 2].
Available from: http://www.rbm.who.int/amd2003/amr2003/ch4. htm

[8] Menendez C. The effect of malaria during pregnancy on infant mortality in an area of low malaria transmission. Am J Trop Med Hyg 2001; 15(5): 459-65.

[9] Mutabingwa TK, LN Malle, de Geus A, Oosting J. Malaria chemosuppression during pregnancy. II: Its effects on maternal haemoglobin levels, placental malaria and birth-weight. Trop Geograph Med 1993; 45: 49-55.

[10] Phillip-Howard P. Epidemiological and control issues related to malaria in pregnancy. Ann Trop Med Parasitol 1999; 93(Suppl 1): 11-7.

[11] Vallely A, Vallely L, Changalucha J, Greenwood B, Chandramohan D. Intermittent preventive treatment for malaria in pregnancy in Africa: what's new, what's needed? Malar J 2007; 6:16.

[12] WHO, UNICEF. Antenatal Care in Developing Countries: Promises, Achievements and Missed Opportunities: Analysis of Trends, levels and differentials. World Health Organization: Geneva 2003.

[13] D'Alessandro U, Brabin B, Verhoeff J. Malaria in pregnancy receives more attention. PREMA-EU Newsletter No. 2: March 2003. [cited 2007 Oct 12]. Available from: http://www.prema-eu.org/

[14] Guyatt HL, Noor AM, Ochola SA, Snow RW. Use of intermittent presumptive treatment and bed nets by pregnant women in four Kenyan districts. Trop Med Int Health 2004; 9(2): 255-61.

[15] Holtz TH, Kachur SP, Roberts JM, Marum LH, Mkandala C, Macheso A. Use of antenatal services and IPT for malaria among pregnant women in Blantyre, Malawi District. Trop Med Int Health 2004; 9(1): 77-82.

[16] WHO: The Abuja Declaration and the Plan of Action: An extract from The African Summit on Roll Back Malaria, Abuja 25 April 2000. WHO/CDC/RBM/2000.17

[17] White NJ. Intermittent Presumptive Treatment for Malaria. PLoS Med 2005; 2(1): e3.

[18] Filler S, Kazembe P, Thigpen M, et al. Randomized trial of 2-dose versus monthly sulfadoxine-pyrimethamine intermittent preventive treatment for malaria in HIV-positive and HIV-negative pregnant women in malawi. J Infect Dis 2006; 194(3): 286-93.

[19] Mutabingwa TK. Antimalarial Intermittent Treatment during Pregnancy in Africa. In: PREMA-EU Newsletter, Issue 1, Nov 2002 [cited 2005 April 14]. Available from: www.prema-eu.org

[20] Greenwood B. The use of antimalarial drugs to prevent malaria in the populations of malaria endemic countries. Am J Trop Med Hyg 2004; 70(1): 1-7.

[21] Verhoeff FH, Brabin BJ, Chimsuku L, Kazembe P, Russel WB, Broadhead RL. An evaluation of effects of intermittent sulfadoxine-pyrimethamine treatment in pregnancy on parasite clearance and risk of low birth-weight in rural Malawi. Ann Trop Med Parasitol 1998; 92(2): 141-50.

[22] EANMAT (East African Network on Malaria Treatment. Monitoring antimalarial drug resistance within national malaria control programmes: the EANMAT experience. Trop Med Int Health 2001; 6(10): 1-8.

[23] Goodman C, Coleman P, Hanson K, Meek S, Mills A. Economics of Malaria Control in sub-Saharan Africa. Global Forum for Health Research, WHO, Geneva 2003.

[24] Mutabingwa TK, Maxwell CA, Sia IG, et al. A trial of proguinildapsone in comparison with sulfadoxine-pyrimethamine for the clearance of Plasmodium falciparum infection in Tanzania. Trans $\mathrm{R}$ Soc Trop Med Hyg 2001; 95: 433-438.

[25] Phillip-Howard PA, Wannemuehler KA, ter Kuile FO, et al. Diagnostic and prescribing practices in peripheral health facilities in rural Kenya. Am J Top Med Hyg 2003; 68 (Suppl 4): 44-49.

[26] Rønn A, Msangeni H, Mhina J, Wernsdorfer W, Bygbjerg I. High level of resistance of $P$. falciparum to sulfadoxine-pyrimethamine in children in Tanzania. Trans R Soc Hyg Trop Med 1996; 54: 179181 .

[27] Menendez C. Strategies for scaling up intermittent presumptive treatment for malaria and anaemia in pregnant women and children. Report of the Scientific Working Group on Malaria, WHO 2003; 83-132.

[28] Goodman C, Coleman P, Mills A. The cost-effectiveness of antenatal prevention in sub-Saharan Africa. Am J Trop Med Hyg 2001; 64(1 Suppl): 45-56.

[29] Murray SF, Nyambo M. Exploring the role of private maternity services in India, Nepal and Tanzania. Final Report. Submitted to 
DFID by the Options Consultancy Services and King's College, London, 2003.

[30] Brieger B. Treating Malaria in Pregnancy: Searching for an Answer. Malaria Matters; 2007 [cited 2007 October 16]. Available from: http://www.malariafreefuture.org/blog/?cat=3

[31] Dellicour S, Hall S, Chandramohan D, Greenwood B. The safety of artemisins during pregnancy: a pressing question. Malar J 2007; 6(1): 15-17.

[32] Anders K, Marchant T, Chambo P, Mapunda P, Reyburn H. Timing of intermittent preventive treatment for malaria during pregnancy and the implementation of current policy on early uptake in north-east Tanzania. Malar J 2008; 7 : 79 .

[33] Mubyazi GM, Bygbjerg IC, Magnussen P, et al. Opportunities, achievements and challenges for scaling-up malaria chemoprevention in pregnancy in Tanzania: perspectives of national level officers. Malar J 2008; 7: 135.

[34] Hill J, Kazembe P. Reaching Abuja Targets for intermittent preventive treatment of malaria in pregnancy in African women: a review of progress and operational challenges. Trop Med Int Health 2006; 11(4): 409-18.

[35] Mbonye AK, Neema S, Magnussen P. Malaria in pregnancy, risk perceptions and care seeking practices among adolescents in Mukono district, Uganda. Int J Adolescent Med Health 2006; 18(4): 561-73.

[36] Mbonye AK. A new approach to deliver malaria prevention interventions to pregnant women at community level in Uganda. PhD Thesis: University of Copenhagen, Denmark 2006.

[37] Ribera MJ, Hausmann-Muela S, D'Alesaandro U, Grietens PK. Malaria in pregnancy: What can social sciences contribute? PLoS Med 2007; 4(4): e92.

[38] Mubyazi G, Bloch P, Byskov J, Hansen K, Olsen Ø, Magnussen P. Economic and other contextual determinants of the acceptability and viability of intermittent preventive treatment of malaria during pregnancy in Tanzania. Acta Tropica MIM Conf Abstracts 2005; 95S(2005): S1-S506.

[39] Breman JG, Alilio MS, Mills A. Conquering the intolerable burden of malaria: What is needed: A summary. Am J Trop Med Hyg 2004; 71(Suppl 2):1-15.

[40] Tanner M, Vlassof C. Treatment seeking behaviour for malaria: a typology based on endemicity and gender. Soc Sci Med 1998; 46: 523-32.

[41] WHO. Implementation research: bridging the gap between efficacy trials and application. TDR Newslett 2004; No. 71.

[42] Hanson K, Goodman C, Lines J, Meek S, Bradley D, Mills A. The economics of malaria control interventions. Global Forum for Health Research: WHO Geneva 2004

[43] Malaria Consortium. Tanzania Roll Back Malaria Consultative Mission Report. Malaria Consortium, London, UK 172004.

[44] Walley J, Khan AM, Shah KS, Witter S, Xiaolin W. How to get practice into research. Bulletin of the WHO 2007; 85(6): 424-5.

[45] Pokhrel S. Scaling up interventions in resource-poor countries: what role does research in stated-preference framework play? A commentary. Health Res Policy Syst 2006; 4: 4.

[46] Mills A. The challenges of scaling up malaria control. Acta Tropica Fourth MIM Pan-African Conf Abstract No. O-75 2005; S47.

[47] Adam YM, Salihu HM. Barriers to antenatal and obstetric care services in rural Kano. J Obstet Gynae 2002; 6(22): 600-3.

[48] Rogerson SL, Chaluluka E, Kanjala M, Mkundika P, Mhango CG, Molyneux ME. Intermittent sulfadoxine-pyrimethamine in pregnancy: effectiveness against malaria morbidity in Blantyre, Malawi, in 1997-1999. Trans R Trop Med Hyg 2000; 94: 549-53.

[49] Eijk AM, Blokland IE, Slutsker L, et al. Use of intermittent preventive treatment for malaria in pregnancy in a rural area of western Kenya with high coverage of insecticide treated bednets. Trop Med Int Health 2005; 10(11): 1134-40.

[50] Robb A. Malaria in pregnancy: Implementation of Interventions. Ann Trop Med Parasitol 2002; 93(1 Suppl 1): 67-70.

[51] Ensor T, Cooper S. Overcoming barriers to health service access and influence on the demand side through purchasing. Health and Nutrition Discussion Paper, International Bank for Reconstruction and Development: Washington DC, September 2004.

[52] Puska P. WHO Director General election: public-health infrastructures: Comment. Lancet 2006; 368: 1400-3.

[53] Magnussen P. Antimalarial intermittent treatment during pregnancy: how do we ensure coverage and compliance in Africa? In:
PREMA-EU Newsletter No 2, pp 4-6, March 2003. [cited 2005 April 14]. Available from: www.prema-eu.org

[54] van Eijk AM, Bles H, Odhiambo F, Ayisi JG, Blokland EI, Rosen DH. Use of antenatal services and delivery among women in rural western Kenya: a community based survey. Reprod Health 2006; 3 : 2.

[55] WHO. Provision of effective antenatal care: Standards for maternal and neonatal care: Doc. No 1.6: Integrated Management of Pregnancy and Childbirth (IMPAC). World Health Organization, Geneva, 2005. [cited 2005 April 14]. Available from: http/www. who.int/making_pregnancy_safer/publications/standards1.6N.pdf

[56] WHO. Realizing the potential of antenatal care. In The World Health Report, 2005. WHO Geneva. [cited 2007 August 12]. Available from: http://www.who.int/whr/2005/chapter3/en/index2. html

[57] Barat LM, Palmer N, Basu S, Worrall E, Hanson K, Mills A. Do malaria control interventions reach the poor? A view through the equity lens. Am J Trop Med Hyg 2004; 71(Suppl 2): 174-8.

[58] Borghi J, Ensor T, Somanathan A, Lissner C, Mills A. Mobilizing financial resources for maternal health. Lancet 2006; 368: 1457-65.

[59] Frederickx I. Health in rural Tanzania: the determinants of health status, health care demand and health care choice: Discussion Paper Series DPS 98.03. Katholieke Universiteit Leuven, Centre for Economic Studies 1998.

[60] Fantahun M, Olwit G. Factors related to Antenatal Clinic Choice and reported activities by pregnant women in Gulele district, Addis Abeba. Ethiop Med J 1995; 33(1): 51-8.

[61] Magadi MA, Madise NJ, Rodrigues RN. Frequency and Timing of Antenatal Care in Kenya: Explaining variations between women in Different Communities. Soc Sci Med 2000; 51: 551-61.

[62] Overbosch GB, Nsowah-Nuamah NNN, van den Boom GJM, Damnyag L. Determinants of antenatal care use in Ghana. J Afr Econ 2004; 13(2): 277-301.

[63] Thaddeus S, Maine D. Too far to walk: maternal mortality in context. Soc Sci Med 1994; 38: 1091-110.

[64] Wagle RR, Sabroe S, Nielsen BB. Socio-economic and physical distance to maternity hospital as predictors for place of delivery: an observation study from Nepal. Pregnancy Child Birth 2004; 4: 8.

[65] Langer A, Villar J, Romero M, et al. Are women providers satisfied with antenatal care? Views on a standard and simplified, evidence-based model of care in four developing countries. BMC Women's Health 2002; $2: 7$.

[66] Acharya LB, Cleland J. Maternal and child health services in rural Nepal. Health Policy Plan 2000; 15(2): 223-9.

[67] Leonard KL, Mliga GR, Mariam HD. Bypassing health centres in Tanzania: revealed preferences for quality. J Afr Econ 2002; 11(4): 441-71.

[68] Nganda RY, Drakeley C, Reyburn H, Marchant T. Knowledge of malaria influences the use of insecticide treated nets but not intermittent presumptive treatment by pregnant women in Tanzania. Malar J 2004; 3: 42.

[69] van Eijk AM, Ayisi AG, tel Kuile FO, et al. Implementation of intermittent preventive treatment with sulfadoxine-pyrimethamine for control of malaria in pregnancy in Kisumu, western Kenya. Trop Med Int Health 2004; 9(5): 630-7.

[70] Priya N. Gender dimensions of user fees: Implications for women's utilisation of health care. Reprod Health Matters 2002; 10(20): 127 34.

[71] Marchant T, Armstrong Schellenberg JRM, Edgar T et al. Anaemia during pregnancy in southern Tanzania. Ann Trop Med Hyg Parasitol 2002; 96(5): 477-87.

[72] Ndyomugyenyi R, Neema S, Magnussen P. The use of informal services for antenatal care and malaria treatment in rural Uganda. Health Policy Plan 1996; 13(1): 94-102.

[73] Kambarami RA, Chirenje MZ, Rusakaniko S. Antenatal care patterns and risk factors associated with perinatal outcome in two rural districts in Zimbabwe. Cent Afr J Med 1999; 45: 294-9.

[74] Okonofua FE, Feyiseteam BJ, Dvies-Adetugbo, Sanusi YO. Influence of socio-economic factors on the treatment and prevention of malaria in pregnant and no-pregnant adolescent girls in Nigeria. $\mathbf{J}$ Trop Med Hyg 1992; 95(5): 309-15.

[75] Wilkinson D, Gouws E, Sach M, Karim SS. Effect of removing user fees on attendance for curative and preventive primary health care services in rural South Africa. Bull WHO Organ 2001; 79(7): $665-71$. 
[76] Mubyazi G, Bloch P, Kamugisha M, Kitua A, Ijumba J. Intermittent preventive treatment of malaria during pregnancy in Tanzania: a qualitative study of knowledge, attitudes and perceptions of district health managers, antenatal service staff and pregnant women in Korogwe district, northeastern Tanzania. Malar J 2005; 1 4: 31

[77] Nyonator F, Kutzin J. Health for some? The effects of user-fees in the Volta Region of Ghana. Health Policy Plan 1999; 14(4): 32941

[78] Mubyazi GM, Mushi A, Kamugisha M, et al. Community views on health sector reform and their participation in health priority setting: case of Lushoto and Muheza districts, Tanzania. J Public Health 2007; 29(2): 147-56.

[79] Boller C, Wyss K, Mtasiwa D, Tanner M. Quality and comparison of antenatal care in private and public providers in Dar Es Salaam, United Republic of Tanzania. Bull WHO 2003; 81(2): 116-22.

[80] Mubyazi G, Massaga J, Kamugisha M et al. Effects of user charges on revenue collections, quality of care and patient attendances for malaria at government dispensaries and health centres in Korogwe district, Tanzania. Health Serv Manage Res 2006; 19(1): 23-35.

[81] Mubyazi GM. The Tanzanian policy on health care fee waivers and exemptions in practice as compared with other developing countries: evidence from recent local studies and international literature. East Afr J Public Health 2004; 1(1): 11-7.

[82] Creel LC, Sass JV, Yinger N. New perspectives on Quality of Care, No 2, 1996; pp1-8. Population Reference Bureau, Washington [cited 2007 June 10]. Available from: http://www.prb.org/pdf/ NewPerspQOC-Clients.pdf

[83] Mbonye AK, Neema S, Magnussen P. Perceptions on use of sulfadoxine-pyrimathamine in pregnancy and the policy implications for malaria control in Uganda. Health Policy 2006; 77(3): 279-89.

[84] Mubyazi GM, Mushi AK, Shayo E, et al. Local primary health care committees and community-based health workers in Mkuranga district, Tanzania: Does the Public Recognise and Appreciate Them? Ethno Med 2007; 1: 27-35.

[85] Murira N, Lutzen K, Lindmark G, Christensson K. Communication patterns between health care providers and their clients at an antenatal clinic in Zimbabwe. Health Care Women Int 2003; 24(2): 8392.

[86] Mufubenga P. Prevention and control of malaria in pregnancy: The Ugandan experience. In: PREMA-EU, Newsletter No. 2, March 2003; pp 6-11. [cited 2005 April 14]. Available from: www.premaeu.org

[87] Fathalla M. Preface. Paediatr Perinat Epidemiol 1988; 12 (Suppl 2): 7-8.

[88] Irshad O. Intermittent treatment of malaria in pregnancy in Somalia. Presentation at the Roll Back Malaria Meeting in Muscat, 2002. [cited 2007 September 17]. Available from: http://www.emro.int/ RBM/Meetings/Muscat02/Presentations

[89] Mwenesi HA. Sociocultural and behavioural issues in the treatment and prevention of malaria. Report of the Scientific Working Group on Malaria, WHO 2003; 126-132.

[90] Nsimba ED. How sulfadoxine-Pyrimethamine was perceived in some rural communities after phasing out chloroquine as a first-line drug for uncomplicated malaria in Tanzania: lessons to learn towards moving from monotherapy to fixed combination therapy. J Ethnobiol Ethnomed 2006; 2: 5

[91] Adams SJ, Broadbent J, Clayden LM, Ridley M. Erythema multiforme (Stevens-Johnson) precipitated by fansidar. Postgrad Med J 1985; 61(713): 263-4.

[92] Lenox-Smith I. Fatal Stevens-Johnson syndrome associated with Fansidar and Chloroquine. J Infect 1987; 14(1): 90-2.

[93] Miller KD, Lobel HO, Satriale RF, Kuritsky JN, Stern R, Campbell CC. Severe cutaneous reactions among American travelers using sulfadoxine-pyrimethamine (fansidar) for malaria prophylaxis. Am J Trop Med Hyg 1986; 35(33): 451-58.
[94] Phillip-Howard PA, Behrens RH, Dunlop J. Steven-Johnson syndrome due to pyrimethamine/sulfadoxine during presumptive self therapy of malaria. Lancet 1989; 2(8666): 803-4.

[95] Bjorkman A, Phillip-Howard PA. Adverse reaction to sulfa drugs: implications for malaria chemotherapy. Bull WHO 1991; 69:297304.

[96] Newman RD, Parise ME, Slutsker L, Nahlen B, Steketee RW. Safety, efficacy and determinants of effectiveness of antimalarial drugs during pregnancy: implications for prevention programmes in Plasmodium falciparum-endemic sub-Saharan Africa. Trop Med Int Health 2003; 8(6): 488-506.

[97] Mubyazi GM, Gonzalez-Block MA. Research influence on national antimalarial drug policy in Tanzania: case study of changing from chloroquine to sulfadoxine-pyrimethamine. Malar J 2005; 4:51.

[98] Mansfield PR, Lexchin J, Wen LS, et al. Educating Health Professionals about Drug and Device Promotion: Advocates' Recommendations. PLoS Med 2006; 3(11): e451.

[99] DCP. Achieving the Millennium Development Goals for Health: So far progress is mixed-can we achieve our targets? Disease Control Priorities Project (DCP). The World Bank Group, 2006 and 2007. [cited 2007 November 10]. Available from: http://www. dcp2.org/pubs/DCP/Section/940

[100] Sachs JD. Achieving the Millennium Development Goals- The Case of Malaria. N Engl J Med 2005; 352(2): 115-7.

[101] Wyss K. An approach to clarifying human resources constraints to attaining health-related Millennium Development Goals. Hum Resour Health 2005; 2: 11 .

[102] Hongoro C, McPake B. Editorial: Human resources in health: putting the right agenda back to the front. Trop Med Int Health 2003; 8(11):965-6.

[103] Mathole T, Lindmark G, Ahlberg BM. Dilemmas and paradoxes in providing and changing antenatal care: a study of nurses and midwives in rural Zimbabwe. Health Policy Plan 2005; 20(6): 385-93.

[104] Mathole T, Lindmark G, Majoko F, et al. A qualitative study of women's perspectives of antenatal care in a rural area of Zimbabwe. Midwifery 2004; 20(2): 122-32.

[105] Worrall E, Morel C, Shunmay Y, et al. The economics of malaria in pregnancy - a review of the evidence and research priorities. Lancet 2007; 7 : 156-68.

[106] Becker $\mathrm{MH}$. The health belief model and personal behaviour Health Educ Monogr 1974; 2(4):409-19.

[107] Family Health International. Behaviour change - A summary of Four Major Theories, 2002. [cited 2007 November 10]. Available from: http://www.fhi.org/NR/rdonlyres/ei26vbslpsidmahhxc332 vwo3g233xsqw22er3vofqvrfjvubwyzclvqjcbdgexyzl3msu4mn6xv5 j/BCCSummaryFourMajorTheories.pdf

[108] Greenwood B, Alonso P, ter Kuile F, Hill J, Steketee R. Malaria in pregnancy: priorities for research. Lancet Infect Dis 2007; 7(2): 169-74.

[109] Baiden F, Baiden R, Williams J, et al. Review of antenatal-linked voluntary counselling and HIV testing in sub-Saharan Africa: Lessons and Options for Ghana. Ghana Med J 2005; 39(1): 8-13.

[110] Schultz L, Steketee RW, Parise M, Wirima JJ, Oloo A, Nahlen B. A selection of Essays: Malaria During Pregnancy: An antenatal Intervention Strategy Whose Time Has Come. [cited 2004 August 26]. Available from: IRDC 2001, http://web.idrc.ca/en/ev/28394201-1-DO_TOPIC.html

[111] van Geertruyden J-P, Thomas F, Erhart A, D'Alesandro U. The contribution of malaria in pregnancy to perinatal mortality. Am J Trop Med Hyg 2004; 71(Suppl 2): 35-40.

[112] WHO. WHO renews call for effective malaria treatment. World Health Organization, 2004; [cited 2007 November 17]. Available from: News-Medical.net, at www.newsmedical.net/ 